\title{
PRIMERA APROXIMACIÓN A LA PALEOENTOMOLOGÍA DE LOS YACIMIENTOS DE LA SIERRA DE ATAPUERCA (BURGOS, ESPAÑA): LA FAUNA SUBFÓSIL DE ORIBÁTIDOS (ACARI, ORIBATIDA)
}

\author{
J. Arroyo ${ }^{1}$, A. de la Riva-Caballero ${ }^{2}$, J. C. Iturrondobeitia ${ }^{3}$, \\ J. M. Bermúdez de Castro ${ }^{4}$, E. Carbonell ${ }^{5}$, J. L. Arsuaga ${ }^{6}$ y C. Díez
}

\begin{abstract}
RESUMEN
Los yacimientos cuaternarios de la Sierra de Atapuerca ofrecen interés mundial por haber hospedado varias especies de homínidos que vivieron en el último millón de años. En este contexto, durante la campaña de excavaciones de 2003 se realizó un muestreo puntual en algunos de sus yacimientos para obtener los primeros datos de restos de artrópodos que pudieran conservarse en los mismos.

En este trabajo se presentan los resultados obtenidos de ácaros subfósiles pertenecientes al suborden de los oribátidos (Acari, Oribatida). Después de procesar los sedimentos, se obtuvieron un total de 7 individuos en el conjunto de los yacimientos muestreados, de los cuales 6 aparecieron en Gran Dolina en un nivel con datación en torno a los 300.000 años. Los ejemplares, identificados en su mayoría a nivel taxonómico de especie, pertenecen a las familias Cosmochthoniidae, Scheloribatidae, Oribatulidae y Hemileiidae. Dado el conocimiento que se dispone de la biología de los taxones encontrados, que pertenecen a géneros y especies presentes en la actualidad, se han realizado inferencias sobre los ambientes pretéritos en que los animales vivieron.
\end{abstract}

Palabras clave: Oribátidos, Cuaternario, Paleoambientes, Atapuerca, España.

\section{SUMMARY}

A first approach to the Paleoentomology present in the Quaternarian sites of Atapuerca (Burgos, Spain): the subfossil oribatid fauna (Acari, Oribatida)

The Atapuerca Quaternarian sites are of worldwide interest due to the presence of human remains belonging to the last million years. The oribatid mites (Acari, Oribatida) found in several archaeological samples extracted from the Atapuerca Quaternarian deposits, have been analyzed during the 2003 excavation campaign to study the

School of Biology and Environmental Science (west). University College Dublin. Belfield Campus, Dublin 4, Ireland.

E-mail: julio.arroyo@ucd.ie (Dr. Julio Arroyo, autor para correspondencia y pruebas)

Bergen Museum, Department of Biology, University of Bergen, Museèplass 3, 5007 Bergen, Norway.

Departamento de Zoología y Biología Celular Animal. Facultad de Ciencia y Tecnología. Universidad del País Vasco (UPV-

EHU). E-48940, Leioa, Vizcaya.

Museo Nacional de Ciencias Naturales (CSIC). Gutiérrez Abascal, 2. 28006 Madrid.

Fac. Lletres. Universitat Rovira i Virgili. Plaza Imperial Tarraco 1. 43005 Tarragona.

ISCIII. c) Sinesio Delgado no 4, Pabellón 14. Madrid 28029.

Area de Prehistoria. I+D+i, Universidad de Burgos, M. Bañuelos, s/n. 09001 Burgos. 
Palaeoentomology of the site. The oribatid mite fauna consists of 7 individuals, 6 of which were obtained from Gran Dolina site (about 300.000 years old) and belong to families Cosmochthoniidae, Scheloribatidae, Oribatulidae and Hemileiidae. Most of the taxa were identified to species level.

The results obtained were used as a basis to reconstruct the paleo-environments of the site in correspondence with the biological and ecological preferences of the taxa.

Key words: Oribatids, Quaternary, Environmental reconstruction, Atapuerca, Spain.

\section{Introducción}

La Sierra de Atapuerca (Burgos, España), es una pequeña elevación de materiales cretácicos dentro del dominio de la cordillera Ibérica, donde se encuentran diferentes depósitos arqueopaleontológicos que cubren buena parte del registro conocido para el Pleistoceno inferior y medio europeo (Carbonell et al., 1999).

Posee un sistema de conductos subterráneos de galerías y dolinas, resultado de la erosión y disolución de las rocas calizas, característico de los sistemas kársticos. Hace cinco millones de años, cuando el río Arlanzón formó su actual valle, el nivel del agua descendió y las cuevas más elevadas se llenaron de aire mientras el valle se hacia más profundo. En este contexto, las galerías se abrieron al exterior y fueron ocupadas por mamíferos, aves, reptiles... y homínidos. Los sedimentos cubrieron posteriormente sus fósiles, testimonio de nuestro pasado y que, en el caso de los homínidos, dan fama mundial a los yacimientos por los excepcionales hallazgos paleoantropológicos obtenidos (Bermúdez de Castro et al., 1997; Arsuaga et al., 1999).

Si bien tanto la Paleoantropología como la Paleobotánica y la Paleozoología de vertebrados son investigados en Atapuerca, el estudio de artrópodos fósiles o Paleoentomología es inédito.

La Paleoentomología estudia los artrópodos fósiles, siendo de gran importancia desde el punto de vista biogeográfico, taxonómico y filogenético (Bukland, 2000). El desarrollo de esta disciplina genera gran información acerca de las condiciones medioambientales y sus cambios en el pasado. La posibilidad de reconstruir a través de los restos de artrópodos los paleoambientes es un campo en auge desde hace décadas, extrapolando las condiciones ecológicas y ambientales (Coope, 1975; Kendward, 1976; Lemdhal, 1988) e incluyendo el tratamiento estadístico de los datos (Buckland et al., 1997; Cong \& Asthworth, 1997).

Dentro de la Paleoentomología, el estudio en yacimientos arqueológicos (Cuaternario) o Arqueoen- tomología se inició en los años 60 como disciplina independiente (Moret, 1996).

El Cuaternario, que incluye al Pleistoceno (1,64-0,01Ma) y el Holoceno, abarca casi los dos últimos millones de años de la historia de la Tierra, período donde se sitúan los yacimientos estudiados en este trabajo, y en el, los artrópodos presentan gran riqueza (Elías et al., 1992).

Dentro de la Arqueoentomología se ha producido el nacimiento de una disciplina, la Arqueoacarología, con los ácaros como protagonistas. Ello es posible dado que grupos como los oribátidos (Oribatida) y los gamásidos (Gamasida) poseen un alto grado de quitinización que les permite, bajo condiciones anóxicas, su conservación en la mayoría de sedimentos geológicos (Solhøy \& Solhøy, 2000).

Los ácaros oribátidos, objeto del presente trabajo, son artrópodos encuadrados en la clase Arachnida, subclase Acari, superorden Acariformes y en el suborden Oribatida o Cryptostigmata. Son muy abundantes en el medio edáfico, de vida libre y sus formas adultas están generalmente bien esclerosadas. Son conocidos desde el Devónico, estando descritas más de 150 familias (Balogh \& Balogh, 1992).

La Arqueoacarología se inició en Europa con Schelvis (1989, 1990, 1992), si bien la Paleoacarología se inició en Escandinavia con Nordenskiöld a principios del siglo $\mathrm{XX}$.

Su desarrollo se estancó hasta principios de los 70 cuando Karppinen y Koponen (de la RivaCaballero, com. pers., 2006) estudiaron oribátidos subfósiles de una turbera finlandesa. A este le siguieron una serie de trabajos en Europa (Karpinnen et al., 1979; Krivolutsky \& Drug, 1986; Schelvis, 1992) y en Estados Unidos (Norton et al., 1988)

Ericsson (1988), inició el uso de oribátidos para inferir ambientes pasados, y Solhøy \& Solhøy (2000) les usaron para la primera reconstrucción ambiental del Holoceno en Europa.

En España, esta disciplina se inicia con los trabajos de Arillo et al. (1992) y de Morales y Sanz 
Tabla 1.- Descripción estratigráfica, cronológica y coordenadas UTM de los yacimientos muestreados.

Table 1.- Stratigraphic and crono-geological description of the sites, together with their UTM coordinates.

\begin{tabular}{lccc}
\hline YACIMIENTO & NIVEL Y CUADRO & UTM & CRONOLOGÍA \\
\hline Covacha de Zarpazos & Nivel TZ III, cuadro Ñ 4- Z 217 & x: 457297, y: 4689132 & Pleistoceno Medio \\
Gran Dolina & Nivel TD 10, cuadro K 21 Z - Z 330 & x: 457275, y: 4689170 & $372 \pm 33$ ka (media ponderada) \\
Sima del Elefante & Nivel TE 14B, cuadro H31 & x: 457337, y: 4688988 & Pleistoceno Inferior \\
Cueva de El Mirador & Nivel 16 techo, cuadro Q 21, facies VL & x: 458346, y: 4688105 & $5700 \pm 70$ \\
\hline
\end{tabular}

(1994). Este tipo de investigaciones ayudan a entender mejor la influencia que los seres humanos ejercen a lo largo de la evolución en el medio que lo rodea.

En general, los ácaros poseen mayor potencial arqueológico que los insectos, pues no necesitan condiciones tan particulares como estos para conservarse (Moret, 1996). Además, los restos cuaternarios, pertenecen generalmente a especies actuales de preferencias biológicas conocidas, lo que permite inferir conclusiones y extrapolar resultados sobre los medios en que vivieron. Así mismo, su gran abundancia en medios naturales y en sedimentos arqueológicos, potencia su capacidad para reconstrucciones ambientales (Sølhøy, 2002).

Por ello, el estudio de los restos de ácaros genera amplia variedad de información para el conocimiento acerca de condiciones ambientales pretéritas en el pasado (Schelvis, 1992), los cambios que se han producido y, posiblemente, cómo se han producido.

El objetivo de este trabajo fue realizar una primera aproximación a la fauna fósil de artrópodos existente en los depósitos cuaternarios de los yacimientos de la Sierra de Atapuerca, tanto en su vertiente taxonómica como en su aplicación paleoambiental.

\section{Área de estudio}

Los yacimientos de la Sierra de Atapuerca se hallan sobre una pequeña elevación situada al centroeste de la provincia de Burgos, que forma parte geográficamente del sector conocido como el Estrecho de Burgos, puente de enlace entre el sistema Ibérico y la cordillera Vasco-Cantábrica. Geológicamente, es una pequeña estructura anticlinal constituida por calizas, arenas y areniscas del Cretácico Medio Superior (80 a 100 m.a.).
Los yacimientos muestreados en este trabajo, (cuadro, estratigrafía, cronología y coordenadas UTM, en Tabla 1) fueron:

- Gran Dolina: antigua cueva que estuvo en tiempos abierta al exterior. En ella se han descubierto los primeros restos fósiles de homínidos pertenecientes a una nueva especie, Homo antecesor Bermúdez de Castro, Arsuaga et al., 1997.

- Covacha de los Zarpazos: perteneciente al complejo kárstico conocido como Galería, contiene un rico registro paleontológico del Pleistoceno medio.

- Sima del Elefante: cuenta con la presencia de los restos arqueológicos más antiguos de la Sierra ( 1.5 m.a.), en forma de industria en piedra.

- Cueva de El Mirador: sitio de formación más reciente que los anteriores, en él se instalan humanos modernos, agricultores y ganaderos, en el Holoceno.

\section{Material y métodos}

La metodología utilizada en Paleoentomología difiere de la empleada en Neoentomología (estudio de los artrópodos actuales) debido a que en Paleoentomología no se estudian artrópodos sino restos y evidencias fósiles (Peñalver, 1996).

En restos de gran antigüedad que dejan huella fósil en roca se requieren métodos especiales de conservación de las muestras como la exfoliación con cincel y martillo, y posterior limpieza y lavado de las huellas fósiles de artrópodos obtenidas.

En el caso concreto del Cuaternario, los depósitos sedimentarios suelen estar poco consolidados y los insectos fósiles no suelen estar mineralizados, consistiendo sus restos en piezas fragmentadas y desarticuladas del exoesqueleto, si bien los quelice- 
Tabla 2.- Muestras, número de especímenes e identificación de los oribátidos en las diferentes zonas.

Table 2.- Samples, number of specimens and species list from Atapuerca sediments.

\begin{tabular}{lcc}
\hline TAXON & $\mathbf{N}^{\mathbf{0}}$ EJEMPLARES & YACIMIENTO \\
\hline $\begin{array}{l}\text { Familia Scheloribatidae, Grandjean, 1933 } \\
\quad \text { Scheloribates cf minifimbriatus Mínguez, Subías } \text { et Ruiz, 1986 }\end{array}$ & 1 & Gran Dolina \\
$\begin{array}{l}\text { Familia Oribatulidae Thor, 1929. } \\
\quad \text { Oribatula tibialis Michael, 1885 }\end{array}$ & 2 & Gran Dolina \\
$\begin{array}{l}\text { Familia Hemileiidae J. Balogh \& P. Balogh, 1984 } \\
\quad \text { Dometorina plantivaga (Berlese, 1895) }\end{array}$ & 2 & Gran Dolina \\
$\begin{array}{l}\text { Oribatida (estado de conservación precario) } \\
\begin{array}{l}\text { Familia Coschmochthoniidae Grandjean, 1947 } \\
\text { Cosmochthonius lanatus (Michael , 1885) }\end{array}\end{array}$ & 1 & Gran Dolina \\
\hline
\end{tabular}

rados (ácaros, escorpiones, arañas...) pueden dejar en roca huellas fósiles.

El muestreo de sedimentos para el estudio de artrópodos fósiles se puede realizar de distintas formas dependiendo del tipo de depósito del que se extraigan. En el caso de las excavaciones arqueológicas por lo general se suelen extraer muestras directamente de los horizontes de los perfiles excavados. Este muestreo debe controlarse estratigráficamente para la datación del material por correlación (Solhøy, 2002).

El método de extracción de artrópodos cuaternarios usado habitualmente en estudios arqueoentomológicos (Schelvis, 1992; Peñalver, 1996) consiste en lavar el material en un cedazo de unos $250 \mathrm{~mm}$ de luz de malla. Es muy importante que los sedimentos estén bien disgregados, ya que los ácaros pueden fácilmente estar ocultos dentro de un grumo de sedimento (Ericsson, 1988; Drouk, 1997). Un procedimiento común para disgregar los sedimentos arcillosos es añadiendo hidróxido de potasio $(\mathrm{KOH})$ al $10 \%$ a la muestra y dejarla reposar durante dos horas a temperatura ambiente, volviendo a lavar la muestra con agua a continuación para eliminar cualquier residuo de KOH (Solhøy \& Solhøy, 2000) y guardándola en alcohol al 70\%. Posteriormente para extraer los artrópodos del residuo orgánico (artrópodos + material vegetal) se pueden utilizar dos técnicas: examen del residuo orgánico bajo la lupa para la extracción de los restos, o el método de flotación, que fue el utilizado en este estudio.

La técnica de flotación requiere el vertido en un recipiente del residuo orgánico, la adición de para- fina o éter de petróleo y la decantación del exceso con agua fría. Posteriormente, el medio orgánico forma una capa en la superficie del agua con el material fósil y después de lavado, los fósiles así recuperados se guardan en alcohol. Es un método clásico de extracción por flotación diferencial en medio orgánico (Kethley, 1991).

La elección de la técnica de flotación para este trabajo se debió principalmente a la gran cantidad de sedimentos recogidos para su análisis, que dificultaba su inspección bajo la lupa. Cada una de las muestras contenía una cantidad aproximada de dos $\mathrm{Kg}$. de material sedimentario que provenía directamente de lo excavado por los investigadores de Atapuerca en los cuadros seleccionados en cada yacimiento (Ver Tabla 1), excepto para los niveles superiores de El Mirador donde se recogieron de los horizontes en el perfil de una cata.

El procesado de los sedimentos se realizó tres veces consecutivas usando éter de petróleo como medio orgánico. Después de la obtención de la fauna fósil y previamente a su identificación, se realizó una cuarta y última extracción empleando aceite de vaselina. Los restos fósiles se conservaron en alcohol al 70\% para su posterior montaje en preparaciones para observación microscópica utilizando el medio de Hoyer (Krantz, 1978). Los restos de ácaros oribátidos hallados han sido identificados al máximo nivel taxonómico posible.

Las series cronológicas de los yacimientos de Atapuerca están publicadas y realizadas con métodos que van desde la resonancia paramagnética electrónica a las series de uranio para los yacimientos pleistocenos (Falgueres et al., 2001), y los yacimien- 

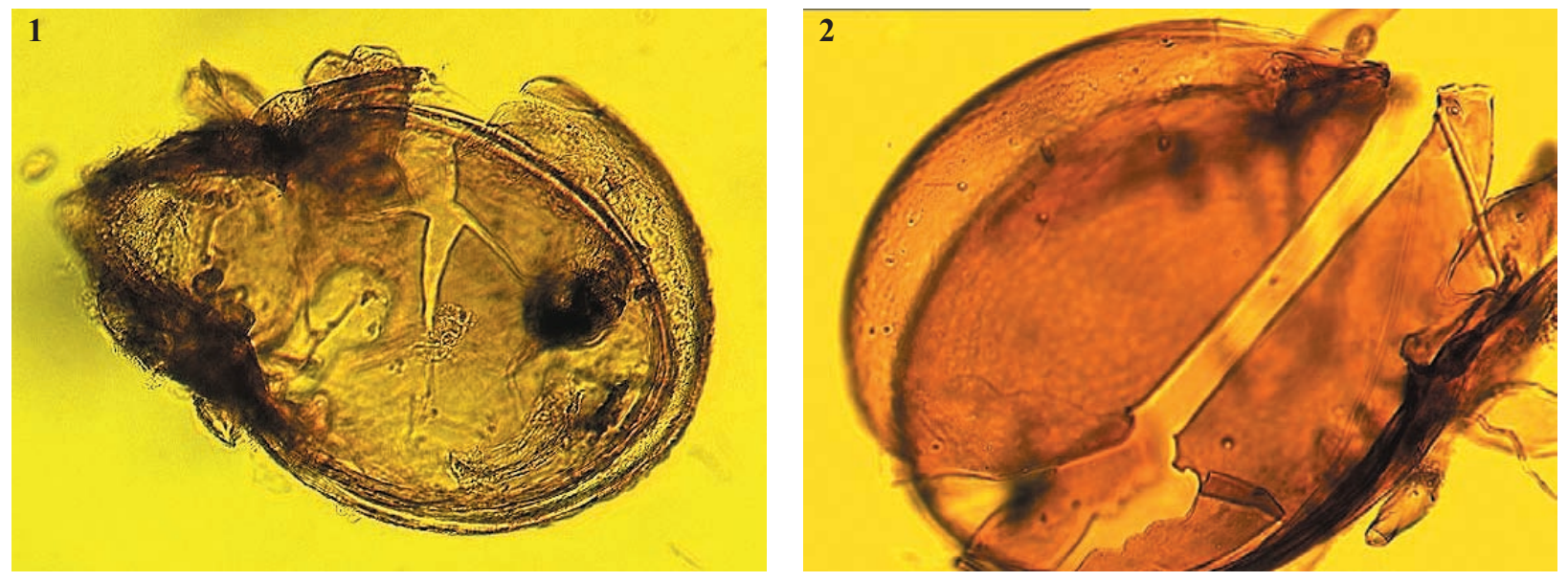

Figs. 1-3.- Individuos subfósiles de ácaros de Atapuerca: 1) Oribatula tibialis Michael, 1885. 2) Dometorina plantivaga (Berlese, 1895). 3) Cosmochthonius lanatus (Michael, 1885). Fotografías: Arroyo, 2007.

Figs. 1-3.- Subfossil individuals of mites from Atapuerca: 1) Oribatula tibialis Michael, 1885. 2) Dometorina plantivaga (Berlese, 1895). 3) Cosmochthonius lanatus (Michael, 1885). Pictures: Arroyo, 2007.

tos holocenos mediante métodos radiocarbónicos convencionales (Vergés et al., en prensa).

\section{Resultados y discusión}

En la tabla 2 se presenta el listado de las especies encontradas con los datos de su abundancia (número de individuos) y yacimiento en el que fueron colectados.

Los ejemplares obtenidos se encuentran depositados temporalmente en el Museo de Ciencias Naturales, Departamento de Entomología de la Universidad de Bergen, Noruega.

A continuación se exponen y discuten los resultados obtenidos.

\section{GRAN DOLINA}

En este yacimiento, aparte de los taxones indicados a continuación, también se encontró un oribáti-

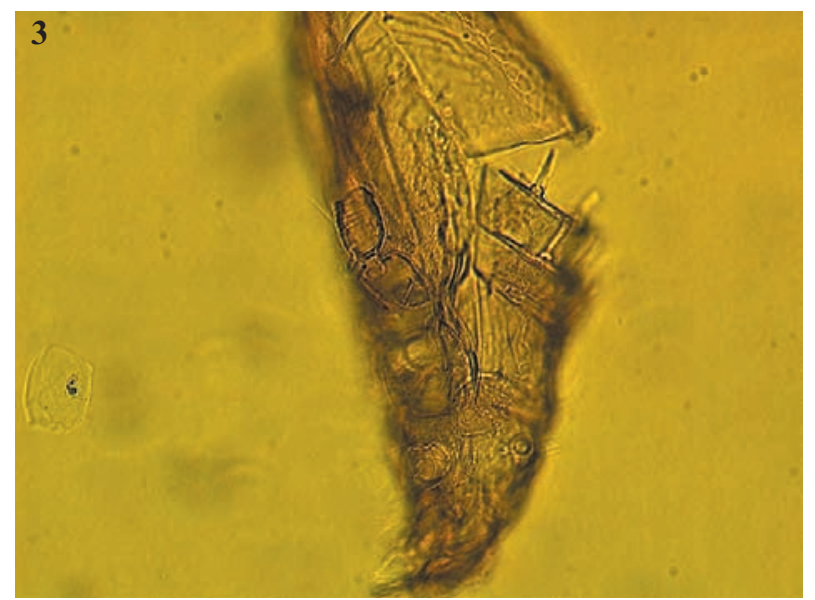

do subfósil que, debido a su precaria conservación, no se pudo identificar.

Familia Scheloribatidae Grandjean, 1933

Scheloribates cf. minifimbriatus Mínguez, Subías et Ruiz, 1986: 1 individuo.

Esta especie es de distribución holártica meridional y ha sido citada en varias ocasiones en la Península Ibérica. Este taxón tiene preferencias xerófilas, no requiriendo ambientes especialmente húmedos (Pérez-Íñigo, 1993). Aparece habitualmente en áreas con vegetación herbácea de tendencias xéricas y también en campos de cultivo.

\section{Familia Oribatulidae Thor, 1929}

Oribatula tibialis Michael, 1885: 2 individuos (Fig. 1).

El género Oribatula contiene especies mayoritariamente cosmopolitas, siendo algunas netamente saxícolas (Pérez-Ĩñigo, 1993). Oribatula tibialis es un 
taxón ubiquista, con una valencia ecológica muy amplia y adaptada a todo tipo de hábitats edáficos lo que dificulta su uso para reconstruir el ambiente donde fosilizaron. Esta especie ha sido frecuentemente hallada en yacimientos cuaternarios en Europa (Karppinen et al., 1979; Schelvis, 1992; Solhøy \& Solhoy, 2000; Larsen et al., 2006).

Familia Hemileiidae J. Balogh \& P. Balogh, 1984

Dometorina plantivaga (Berlese, 1895): 2 individuos (Fig. 2).

Esta especie se caracteriza por presentarse casi exclusivamente en hábitats arborícolas. Su hallazgo en perfiles edáficos es excepcional (Pérez-Íñigo, 1993).

En yacimientos cuaternarios es relativamente frecuente la aparición de especies de la familia Hemileiidae, en concreto la especie encontrada en este estudio ha sido citada tanto en trabajos de Paleoacarología (Larsen et al., 2006) como en trabajos de Arqueoacarología (de la Riva-Caballero, com. pers., 2007) realizados en Escandinavia.

\section{Cueva de El Mirador}

Familia Coschmochthoniidae Grandjean, 1947

Cosmochthonius lanatus (Michael, 1885): 1 individuo (Fig. 3).

Especie de amplia distribución ( $\mathrm{Vu}$ \& Nguyen, 2000), citada en diversos medios edáficos como matorrales, bosques y cultivos. Es la primera vez que se encuentra en un yacimiento cuaternario en la Península, y parece ser la primera cita mundial de la especie como subfósil. Esta falta de registros previos puede deberse al hecho de que al ser un miembro de los Enarthronotra tiene el exoesqueleto opistosómico constituido por diversas placas generalmente menos esclerotizadas, que normalmente se disgregan y es, por tanto, imposible identificar el subfósil encontrado. La escasa antigüedad de El Mirador "facilitaría" la conservación de este género, debido a que su débil esclerotización hace mas improbable su conservación en sedimentos de más edad.

\section{Covacha de los Zarpazos y Sima del Elefante}

No se encontraron oribátidos.
Aunque el número de individuos y de especies encontradas no permite realizar una reconstrucción temporal fiable, sí se puede presentar a grandes rasgos una idea básica del tipo de ambiente que había en torno a Gran Dolina hace 370.000 años.

La cueva de la Gran Dolina se abrió al exterior hace unos 900.000 años (nivel TD4) (Arsuaga \& Martínez, 2005). Este yacimiento presenta fósiles muy bien conservados de diferentes especies animales y en él se descubrió al Homo antecessor, la nueva especie que demuestra la existencia de homínidos en Europa durante el Pleistoceno inferior (Bermúdez de Castro et al., 1997).

En el caso concreto del nivel estudiado en este trabajo (TD10), que está datado en torno a los 370.000 años, se advierte un intenso uso y ocupación de la cavidad por la cantidad de restos líticos y de fauna que aparecen. Haciendo una revisión de los hábitats que normalmente ocupan las especies de oribátidos encontradas en el yacimiento en el nivel muestreado (TD 10), parece que la vegetación del área era arbórea o por lo menos presentaba una cobertera vegetal con presencia de plantas arbustivas o incluso de comunidades vegetales de mayor porte. Como dato de apoyo a estas inferencias, en el nivel TD10 se han descubierto diversos restos de herbívoros, tales como cuernas y huesos de cérvido (Cervus elaphus Linnaeus, 1758 y Dama dama Linnaeus, 1758) (Made, 2001). C. elaphus existe actualmente en la mayor parte de los sistemas de la Península que cuenten con praderas junto a cobertura vegetal leñosa, al menos como refugio, desde llanuras a nivel del mar hasta áreas de alta montaña. Su hábitat preferencial son los ecotonos entre zonas boscosas o cubiertas con vegetación arbustiva y áreas abiertas donde exista producción de plantas herbáceas (Carranza, 2004). También D. dama ocupa hábitats boscosos.

Quizá la principal conclusión es que se confirma la presencia de ácaros subfósiles en los yacimientos de Atapuerca, tal como se pudiera prever dado los trabajos previos realizados en otros yacimientos cuaternarios en relación con este grupo de artrópodos. De la misma forma se puede ofrecer una idea del potencial de estos arácnidos en reconstrucciones paleoambientales. Cabe destacar que ninguna de las especies encontradas de oribátidos en Atapuerca apareció en los muestreos realizados en yacimientos arqueológicos (cuevas y turberas) de la provincia de Lugo (Arillo et al., 1992) de edades comprendida desde 34800 BP hasta la actualidad, aunque la mayoría de individuos aparecieron fundamentalmente en muestras de escasa antigüedad principalmente turberas, y la especie más abundan- 
te con diferencia fue Limnozetes cf onandaga que es un oribátido estrictamente dulceacuícola, lo que explica su abundancia en estas muestreas de condiciones pretéritas que parecen, en principio, diferir de las existentes en los yacimientos que se muestrearon en Atapuerca, tal como se ha comentado al principio de este apartado.

Podemos concluir también que el número de oribátidos subfósiles colectados (alguno de los cuales es de aparición habitual en estudios realizados en otros países) es bajo, dado la puntualidad del muestreo, por lo que es necesario una investigación más profunda en próximas campañas para confirmar y ampliar estos primeros resultados, y establecer extrapolaciones más sólidas sobre el medio ambiente en que vivieron.

\section{AGRADECIMIENTOS}

Deseamos manifestar nuestro agradecimiento a Torstein Solhøy (Noruega) y al Dr. Morales (España) por su ayuda en las consultas planteadas. Asimismo también mostrar nuestro agradecimiento a los distintos investigadores de los yacimientos de la Sierra de Atapuerca por las facilidades y disposición mostrada para la realización de este estudio, así como a los evaluadores de Graellsia por sus valiosas sugerencias y correcciones. Las investigaciones entomológicas de Atapuerca se realizaron dentro del Proyecto BOS2003-08938-C03-02.

\section{Referencias}

Arillo, A., Gil-Martín, J. \& Subías, L. S., 1992. Acaros oribátidos subfósiles de Galicia. Actas do Congresso Iberico de Entomologia, 5(2): 491-498.

Arsuaga, J. L., Lorenzo, C., Carretero, J. M., Gracia, A., Martínez, I., García, N., Bermúdez de Castro, J. $\mathrm{M}^{\mathrm{a}}$. \& Carbonell, E., 1999. A complete human pelvis from the Middle Pleistocene of Spain. Nature, 399: 255-258

Arsuaga, J. L. \& Martínez, I., 2005. Atapuerca y la Evolución Humana. Fundació Caixa Catalunya. Barcelona. 155 pp.

Balogh, J. \& Balogh, P., 1992. The Oribatid Mites Genera of the World. The Hungarian Natural History Museum. Budapest. 263 pp. (vol. I), 375 pp. (vol. II).

Bermúdez de Castro, J. Mª., Arsuaga, J. L., Carbonell, E., Rosas, A., Martínez I. \& Mosquera, M., 1997. A Hominid from the Lower Pleistocene of Atapuerca, Spain: Possible Ancestor to Neandertals and Modern Humans. Science, 276: 1392-1395.

BuckLand, P., 2000. An introduction to Palaeoentomology in Archeology and the BUGS Database Management System. Umea Universitet. Sweden. 62 pp.
Buckland, P. I., Yuan Zhuo, D. \& Buckland, P., 1997. Towards an expert system in Palaeoentomology. In: A. C. Ashworth, P. Buckland \& J. P. Sadler (eds.). Studies in Quaternary Entomology-An Inordinate Fondness for Insects. Quaternary Proceedings, 5: 71-79.

Carbonell, E., Bermúdez de Castro, J. M M $^{\mathrm{a}}$ \& Arsuaga, J. L., 1999. Gran Dolina Site: TD6 Aurora Stratum (Burgos, Spain). Journal of Human Evolution, 37(3/4): 309-700.

CARRAnZA, J., 2004. Ciervo - Cervus elaphus. En: L.M. Carrascal \& A. Salvador (eds.). Enciclopedia Virtual de los Vertebrados Españoles. Museo Nacional de Ciencias Naturales, Madrid. http://www.vertebradosibericos.org/

Cong, S. \& Ashworth, A. C., 1997. The use of Correspondence Analysis in the Analysis of Fossil Beetle Assemblages. In: A. C. Asworth, P. Buckland \& J. P. Sadler (eds.). Studies in Quaternary Entomology. An Inordinate fondness for Insects. Quaternary Proceedings, 5: 79-82.

Coope, G. R., 1975. Climatic fluctuations in northwest Europe since the Last Interglacial, indicated by fossil assemblages of Coleoptera. In: A. E. Wright \& F. Moseley (eds.). Ice Ages: Ancient and Modern, (Geological Journal Issue 6), Seel House Press. Liverpool: 153-168.

Drouk, A. Y., 1997. Acarological Analysis: Problems of Palaeoecological Reconstructions. In: M. E. Edwards, V. Sher \& R. D. Guthrie (eds.). Terrestrial Palaeoenvironmental Studies in Beringia. Alaska Quaternary Center, University of Alaska Museum. Fairbanks: 91-97.

Elías, S. A., Mead, J. I. \& Agenbroad, L. D.,1992. Late Quaternary arthropods from the Colorado Plateau, Arizona and Utah. The Great Basin Naturalist, 52: 59-67.

ERICSSON, J. M., 1988. Fossil oribatid mites as tools for quarternary paleoecologists: preservation quality, quantities, taphonomy. In: R. S. Laub, N. G. Miller \& D. W. Steadman (eds.). Late pleistocene and early holocene paleoecology and archeology of the eastern great lakes region. The Buffalo Society of Natural Sciences, 33: 207-226.

Falgueres, C., Bahain, J. J., Yokoyama, Y., Bischoff J. L., Arsuaga, J. L., Bermúdez de Castro, J. M., Carbonell, E. \& Dolo, J. M., 2001. Datation par RPE et U-Th des sites pléistocenes d'Atapuerca: Sima de los Huesos, Trinchera Dolina et Galería. Bilan géochronologique. L'Anthropologie, 105: 7181.

Karpinnen, E., Krivolutksi, D. A., Kopponen, M., Kozlovskaja, L. S., Laskova, L. M. \& VittasaARI M., 1979. List of subfossil oribatid mites (Acarina, Oribatei) of northern Europe and Greenland. Annales Entomolgicae Fennici, 45(4): 103-108. 
KENWARD, H. K., 1976. Reconstructing ancient ecological conditions from insects remains: some problems and an experimental approach. Ecological Entomology, 1: $7-17$.

Kethley, J., 1991. A procedure for extraction of microarthropods from bulk soil samples with emphasis on inactive stages. Agriculture, Ecosystems and Environment, 34: 193-200.

Krantz, G. W., 1978. A manual of Acarology. Oregon State University Book Stores. Corvallis. 509 pp.

Krivolutski, D. A. \& DruK, A. Y., 1986. Fossil Oribatid mites. Annual Review of Entomology, 31: 533-545.

Larsen, J., Buune, A. E., \& Riva-CAballero, A. D. L., 2006. Holocene Environmental and Climate History of Trettetjørn, a Low-alpine Lake in Western Norway, Based on Subfossil Pollen, Diatoms, Oribatid Mites, and Plant Macrofossils. Arctic, Antarctic and Alpine Research, 38: 571-583.

Lemdhal, G., 1988. Palaeoclimatic and palaeocological studies of subfossil insects from late Weichsehnan sediments in southern Sweden. Lund University, LUNDQUA thesis, nr. 22. Lund. Various paging.

MADE, J. VAN DER, 2001. Les ongules d'Atapuerca. Stratigraphie et biogéographie. L'Anthropologie, 101: 95-113.

Morales, A. \& Sanz, J. L., 1994. Arqueo-acarología: potencialidades y limitaciones de una prácticamente inédita subdisciplina arqueozoológica. Pyrenae, 25: 17-29.

Moret, R., 1996. Arqueo-entomología: cuando los insectos contribuyen al conocimiento de nuestro pasado. Boletín de la Sociedad Entomológica Aragonesa, monográfico "Paleoentomología", 16: 183-188.

Norton, R. A., Bonamo, P. M., Grierson, J. D. \& SHEAR, W. A., 1988. Oribatid mite fossils from a terrestrial Devonian deposit near Gilboa, New York. Journal of Paleontology, 62: 259-269.

Peñalver, R.,1996. Técnicas y métodos de obtención, preparación, conservacion y estudio de insectos fósiles. Boletín de la Sociedad Entomológica Aragonesa, monográfico "Paleoentomología", 16: 157-174.

Pérez-ÍñIgo, C., 1993. Acari, Oribatei, Poronota. En: M. A. Ramos et al. (eds.). Fauna Ibérica, vol. 3. Museo Nacional de Ciencias Naturales, CSIC. Madrid. 320 pp.

Schelvis, J., 1989. Mites (Acari) from the late Neolithic well at Kolhorn (The Netherlands). Palaeohistoria, 31: 165-171.
ScHELVIS, J., 1990. The reconstruction of local environments on the basis of remains of Oribatid mites (Acari; Oribatida). Journal of Archaeological Science, 17: 559-571.

Schelvis, J., 1992. Mites and Archaeozoology. General Methods; Applications to Dutch Sites. Ph.D. thesis, University of Groningen. Rijksuniversiteit Groningen. Groningen. 116 pp.

Solnøy, I. \& Solnøy, T., 2000. The fossil oribatid mite fauna (Acari: Oribatida) in late glacial and early Holocene sediments in Krakenes Lake, western Norway. Journal of Paleoentomology, 23: 35-47.

Solnøy, T., 2002. Tracking environmental change using lake sediments In: J. P. Smol, H. J. B. Birks \& W. M. Last (eds.). Zoological Indicators vol. 16. Kluwer Academic Publishers. Dordrecht: 101-104.

Vergés, J. M., Allué, E., Angelucci, D., Burjachs, F., Carrancho, A., Cebriá, A., Exposito, I., Fontanals, M., Moral, S., Rodríguez, A. \& Vaquero, M., En prensa. Los niveles neolíticos de la cueva de El Mirador (Sierra de Atapuerca, Burgos): Nuevos datos sobre la implantación y el desarrollo de la economía agropecuaria en la Submeseta Norte. IV Congreso Neolitico Peninsular. Alicante 2006.

Vu, Q. M. \& NGuyen, T. T., 2000. Microarthropod community structures (Oribatei and Collembola) in Tam Dao National Park, Vietnam. Journal of Biosciences, 25(4): 379-386.
Recibido, 29-I-2007 Aceptado, 18-V-2007 Publicado, 27-VI-2007 